\title{
Photon correlations in a two-site non-linear cavity system under coherent drive and dissipation
}

\author{
Sara Ferretti and Lucio Claudio Andreani \\ Dipartimento di Fisica "Alessandro Volta", Università di Pavia, via Bassi 6, I-27100 Pavia, Italy \\ Hakan E. Türeci \\ Institute for Quantum Electronics, ETH Zurich, 8093 Zurich, Switzerland \\ Dario Gerace \\ Dipartimento di Fisica "Alessandro Volta", and UdR CNISM, \\ Università di Pavia, via Bassi 6, I-27100 Pavia, Italy
}

(Dated: July 27, 2021)

\begin{abstract}
We calculate the normalized second-order correlation function for a system of two tunnel-coupled photonic resonators, each one exhibiting a single-photon nonlinearity of the Kerr type. We employ a full quantum formulation: the master equation for the model, which takes into account both a coherent continuous drive and radiative as well as non-radiative dissipation channels, is solved analytically in steady state through a perturbative approach, and the results are compared to exact numerical simulations. The degree of second-order coherence displays values between 0 and 1 , and divides the diagram identified by the two energy scales of the system - the tunneling and the nonlinear Kerr interaction - into two distinct regions separated by a crossover. When the tunneling term dominates over the nonlinear one, the system state is delocalized over both cavities and the emitted light is coherent. In the opposite limit, photon blockade sets in and the system shows an insulator-like state with photons locked on each cavity, identified by antibunching of emitted light.

PACS numbers: 42.50.Ar, 42.50.Pq, 71.36.+c, 73.43.Nq
\end{abstract}

\section{INTRODUCTION}

Recent advances in cavity quantum electrodynamics (CQED) have led to the demonstration of a number of striking phenomena related to the fundamental properties of light-matter coupling, e.g. when single or few quantum emitters interact with the mode of an electromagnetic resonator ${ }^{[1]}$ The experimental realization of the Jaynes-Cummings (JC) model ${ }^{4}$ which predicts a strong light-matter coupling regime when the Rabi frequency between the oscillators exceeds their respective loss rates,$\sqrt[566]{6}$ has been achieved in both atomic ${ }^{1}$ and solidstate ${ }^{718}$ CQED with single two-level emitters in high-Q resonators. In the strong coupling regime, the CQED system is intrinsically anharmonic at the level of single quanta, which derives from the underlying anharmonic nature of the emitter's eigenstates. [9] 14

Following these early works, the ultimate limit of nonlinear optics, i.e. the ability to control the nonlinear response of a system by the injection of single photons through the so called photon blockade effect, $\frac{15}{15}$ has been recently reached. Inhibition of the resonant transmission of a single photon because of the presence of another one within the cavity has been experimentally demonstrated with both single atoms in optical cavities ${ }^{16}$ and semiconductor quantum dots strongly coupled to photonic nanocavities ${ }^{17}$ In all these experiments, the statistical properties of the resonant light beam transmitted through the nonlinear system gives precise information on the nature of the effective photon-photon interaction within the cavity. Photon blockade has been shown to be strictly characterized by conversion of a classical, coherent field at the input into a nonclassical, antibunched photon stream at its output ${ }^{15}$ Similar effects have been also predicted for other types of coherent fields $\frac{18}{20}$ More recently, there has been an intense effort towards the exploitation of nonlinearities arising from Coulomb interaction in confined electron and photon systems. ${ }^{21} \mathrm{In}$ such a case, mixed light-matter states (polaritons) arise from the strong coupling regime of quantum well excitons and microcavity photons, which are three-dimensionally confined thanks to the progress in lithographic techniques. The polariton quantum blockade has been predicted for such highly nonlinear light-matter states,, 22 and first evidences of nonlinear behavior have been reported experimentally ${ }_{[23}^{23}$ These systems are likely to provide a further playground for single-photon nonlinear optics in the near future.

Motivated by the great level of control achieved in CQED experiments with single cavities, recent theoretical work has explored multi-cavity nonlinear systems. Initial work was mainly aimed at studying the superfluidinsulator quantum phase transition 24 26 of the effective Bose-Hubbard hamiltonian, $27 \sqrt{32}$ or the JC-Hubbard mode $[33 \sqrt[39]{39}$ for arrays of CQED systems under quasiequilibrium conditions. Subsequent work dealing with coupled non-linear cavity systems has addressed the dynamics in a two-site JC model,$\stackrel{40 \mid 41}{,}$ soliton physics $\stackrel{42}{,}$ a proposal for observing fractional quantum Hall states, $\stackrel{43}{43}$ the possible realization of a Tonks-Girardeau gas in different one-dimensional geometries, $\stackrel{44 / 45}{t}$ the study of effective spin model $\sqrt{46 / 47}$ and of entanglement generation,, 48 
the use of coupled cavity systems as efficient singlephoton sources even in the presence of weak photon nonlinearities $\frac{49}{49}$ and the signatures of superfluidinsulator quantum phase transition for an infinite CQED array under pulsed coherent driving. 50$]$

In a recent work, $\frac{51}{1}$ a proposal has been made to observe signatures of strong photon correlations in a system of three coupled nonlinear cavities, with the central one displaying single-photon nonlinearity, through the measurement of its degree of second order coherence. Besides being a readily realizable system with state-of-the art technology with both atomic and solid state CQED, $[11152[53$ this system is a possible quantum photonic device in which information encoded in classical field states can be controlled by the presence or absence of single photon quanta (a single-photon transistor).

In order to extend and generalize the latter work, here we present a systematic theoretical analysis of a model of two coupled cavities, both of them assumed to be nonlinear at the single photon level. The model takes into account coherent driving as well as global dissipation channels within a master equation treatment (see a sketch of the system, Fig. 1). The dynamical equilibrium reached by the system is due to the balance between pumped and dissipated photons and it is analyzed in steady state. Following previous work, $\frac{51]}{5 e}$ mainly concentrate on calculating the second-order correlation function for the light emitted from each cavity, both analytically and numerically. Interplay of coherent tunneling and on-site interactions is clearly identified in the crossover from Poissonian to sub-Poissonian light statistics of the emitted light.

The paper is organized as follows: we first introduce the model and the master equation in Sec. II. In Sec. III we provide a description of the analytical solution for the master equation that can capture the second-order correlation function for this model, and compare it to a full numerical solution.

\section{THEORETICAL FRAMEWORK}

We will investigate here the photon correlations in the two-site Kerr-Hubbard model (Fig. 1) given by $(\hbar=1)$

$$
\begin{aligned}
\hat{H}_{\mathrm{KH}} & =\sum_{i=1,2}\left[\omega_{i} \hat{p}_{i}^{\dagger} \hat{p}_{i}+U_{i} \hat{p}_{i}^{\dagger} \hat{p}_{i}^{\dagger} \hat{p}_{i} \hat{p}_{i}+F_{i} e^{-\mathrm{i} \omega_{\mathrm{L}} t} \hat{p}_{i}^{\dagger}+F_{i}^{*} e^{\mathrm{i} \omega_{\mathrm{L}} t} \hat{p}_{i}\right] \\
& +J\left(\hat{p}_{1}^{\dagger} \hat{p}_{2}+\hat{p}_{2}^{\dagger} \hat{p}_{1}\right)
\end{aligned}
$$

where $\hat{p}_{i}\left(\hat{p}_{i}^{\dagger}\right)$ destroy (create) generic bosonic excitations in each of the two cavities at their fundamental frequencies $\omega_{i}, U_{i}$ is the nonlinear Kerr-type interaction in each cavity, $J$ is the inter-cavity tunneling rate, and $F_{i} e^{-\mathrm{i} \omega_{\mathrm{L}} t}$ is the coherent driving amplitude at laser frequency $\omega_{\mathrm{L}}$.

There are several ways to realize the model in Eq. (1), with either atomic or solid-state cavity QED technology. All these realizations principally rely on the formation of well-defined quasi-particles, polaritons, of mixed lightmatter nature, for the availability of the required effective

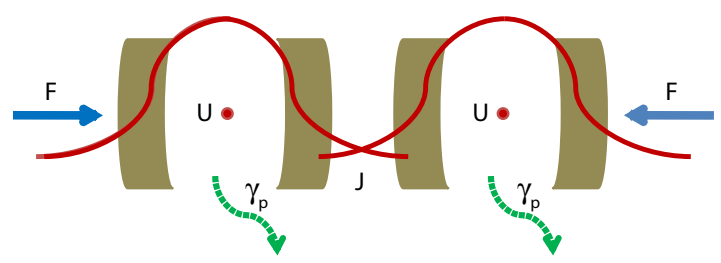

Figure 1: (Color online) The system of two coupled nonlinear cavities. The relevant parameters of the model are indicated, namely the coherent pumping $(F)$ and dissipation $\left(\gamma_{p}\right)$ rates, respectively. The cavities are supposed to be nonlinear, with an interaction energy $U$, and they are tunnel-coupled by evanescent overlap of their cavity modes with a coupling constant $J$. We assume symmetric cavity parameters in this work.

quasi-particle interactions $U_{i}$. One possible way is, e.g., to start from two atoms or quantum dots strongly coupled to their respective cavity modes, with the two cavities in optical contact with each other. While the singlephoton nonlinearity derives from the light-matter coupling in the framework of a JC model, the tunnel coupling is due to photon tunneling and the coherent pump acts directly on the photonic degrees of freedom. In the absence of losses or spontaneous emission decay, such a system would be described by a two-site JC-Hubbard Hamiltonian, extensively discussed in recent literature ${ }^{33 \mid 37 ! 41} \mathrm{In}$ the weak pumping limit and the dispersive regime, the JC nonlinearity can be reduced to an effective Kerr-type nonlinearity between polaritons $\frac{[54}{}$

A more straightforward and conceptually simple way of obtaining a Kerr-type nonlinearity that is effective at the single photon/polariton level is to consider solidstate systems in which the Coulomb interaction is strong enough. In particular, we refer here to excitons in quantum wells coupled to a single photonic mode of a microresonator where excitons interact via their dipole field.22 In such a case, the Hamiltonian for the two-site system in the rotating wave and electric dipole approximations 1) is given by $\hat{H}=\hat{H}_{0}+\hat{H}_{1}$ with $\underline{49}$

$$
\begin{aligned}
\hat{H}_{0} & =\sum_{i=1,2}\left[\omega_{\text {cav }, i} \hat{a}_{i}^{\dagger} \hat{a}_{i}+\omega_{\mathrm{x}, i} \hat{X}_{i}^{\dagger} \hat{X}_{i}+\Omega_{i}\left(\hat{a}_{i}^{\dagger} \hat{X}_{i}+\hat{a}_{i} \hat{X}_{i}^{\dagger}\right)\right](2) \\
\hat{H}_{1} & =\sum_{i=1,2}\left[V_{i} \hat{X}_{i}^{\dagger} \hat{X}_{i}^{\dagger} \hat{X}_{i} \hat{X}_{i}+E_{i}(t) e^{-\mathrm{i} \omega_{\mathrm{L}} t} \hat{a}_{i}^{\dagger}+E_{i}^{*}(t) e^{\mathrm{i} \omega_{\mathrm{L}} t} \hat{a}_{i}\right] \\
& +j\left(\hat{a}_{1}^{\dagger} \hat{a}_{2}+\hat{a}_{2}^{\dagger} \hat{a}_{1}\right) .
\end{aligned}
$$

Here, $\hat{a}_{i}^{\dagger}\left(\hat{a}_{i}\right)$ creates (destroys) a photon in cavity $i$ at frequency $\omega_{\text {cav }, i}$, while the operators $\hat{X}_{i}\left(\hat{X}_{i}^{\dagger}\right)$ describe excitonic quasi-particles with energy $\omega_{\mathrm{x}, i}$. We assume 
an interaction energy $V_{i}$ between excitons deriving from a contact-type Coulomb interaction, and the excitonphoton interaction strength is given by the Rabi frequency $\Omega_{i}$. Cavity photons are coherently pumped into each cavity with amplitudes $E_{i} e^{-\mathrm{i} \omega_{\mathrm{L}} t}$, and $j$ is the tunneling amplitude for photons between neighboring cavities.

With respect to the latter model, polaritonic excitations can be defined as linear combination of excitons and cavity photons as

$$
\left(\begin{array}{c}
\hat{P}_{-, i} \\
\hat{P}_{+, i}
\end{array}\right)=\left(\begin{array}{cc}
u_{i} & -v_{i} \\
v_{i} & u_{i}
\end{array}\right)\left(\begin{array}{c}
\hat{X}_{i} \\
\hat{a}_{i}
\end{array}\right)
$$

where the coefficients are

$$
u_{i}=\frac{1}{\sqrt{1+\left(\frac{\Omega_{i}}{\omega_{-, i}-\omega_{\mathrm{cav}, i}}\right)^{2}}} ; v_{i}=\frac{1}{\sqrt{1+\left(\frac{\omega_{-, i}-\omega_{\mathrm{cav}, i}}{\Omega_{i}}\right)^{2}}} .
$$

This transformation then diagonalizes $\hat{H}_{0}$

$$
\hat{H}_{0}=\sum_{\sigma= \pm} \sum_{i=1,2} \omega_{\sigma, i}\left(\hat{P}_{\sigma, i}\right)^{\dagger} \hat{P}_{\sigma, i}
$$

with the lower and upper polariton energies respectively given by $\omega_{ \pm, i}=\left(\omega_{\text {cav }, i}+\omega_{\mathrm{x}, i}\right) / 2 \pm \sqrt{\Omega_{i}^{2}+\left(\Delta_{i} / 2\right)^{2}}$, where $\Delta_{i}=\omega_{\text {cav }, i}-\omega_{\mathrm{x}, i}$. Assuming quasi-resonant pumping of the lower polariton level $\left(\omega_{\mathrm{L}} \sim \omega_{-}\right), \Delta_{i}<0$ and neglecting non-resonant contributions, the resulting effective Hamiltonian $\hat{H}$ can be written in the form of Eq. (1) with $\hat{p}_{i}=\hat{P}_{-, i}, \omega_{i}=\omega_{-, i}, F_{i}=v_{i} E_{i}, J=v_{1} v_{2} j$ and $U_{i}=u_{i}^{4} V_{i}$. Throughout this work we will be assuming identical sites for simplicity and drop the site indices on the parameters of the system.

Losses resulting, e.g., from spontaneous emission decay of the excitons or cavity photon leakage can be taken into account within the quantum Master equation in the Born-Markov approximation for the density matrix of the quasi-particles in the system, which is expressed in the usual Lindblad form

$$
\frac{\partial}{\partial t} \rho=\mathrm{i}\left[\rho, \tilde{H}_{\mathrm{KH}}\right]+\mathcal{L}(\rho)
$$

where $\tilde{H}_{\mathrm{KH}}$ is the Kerr-Hubbard Hamiltonian written in the rotating frame with respect to the pump frequency resonant with the lower polariton mode $\left(\omega_{-}-\omega_{\mathrm{L}}=0\right)$

$$
\begin{aligned}
\tilde{H}_{\mathrm{KH}} & =\hat{\mathrm{R}}(t) \hat{H}_{\mathrm{KH}} \hat{\mathrm{R}}^{\dagger}(t)=\sum_{i=1,2}\left[U \hat{p}_{i}^{\dagger} \hat{p}_{i}^{\dagger} \hat{p}_{i} \hat{p}_{i}+F \hat{p}_{i}^{\dagger}+F^{*} \hat{p}_{i}\right] \\
& +J\left(\hat{p}_{1}^{\dagger} \hat{p}_{2}+\hat{p}_{2}^{\dagger} \hat{p}_{1}\right)
\end{aligned}
$$

with $\hat{\mathrm{R}}(t)=\exp \left\{\mathrm{i} \omega_{\mathrm{L}}\left(\hat{p}_{1}^{\dagger} \hat{p}_{1}+\hat{p}_{2}^{\dagger} \hat{p}_{2}\right) t\right\}$. The Liouvillian can be expressed in the usual Lindblad form 56

$$
\mathcal{L}=\frac{\gamma_{p}}{2} \sum_{i=1,2}\left[2 \hat{p}_{i} \rho \hat{p}_{i}^{\dagger}-\hat{p}_{i}^{\dagger} \hat{p}_{i} \rho-\rho \hat{p}_{i}^{\dagger} \hat{p}_{i}\right]
$$

where $\gamma_{p}$ is the polariton dissipation rate in each cavity $\left[\frac{57}{}\right.$ We will discuss possible realistic implementation of this model and the required tolerances in Sec. IV

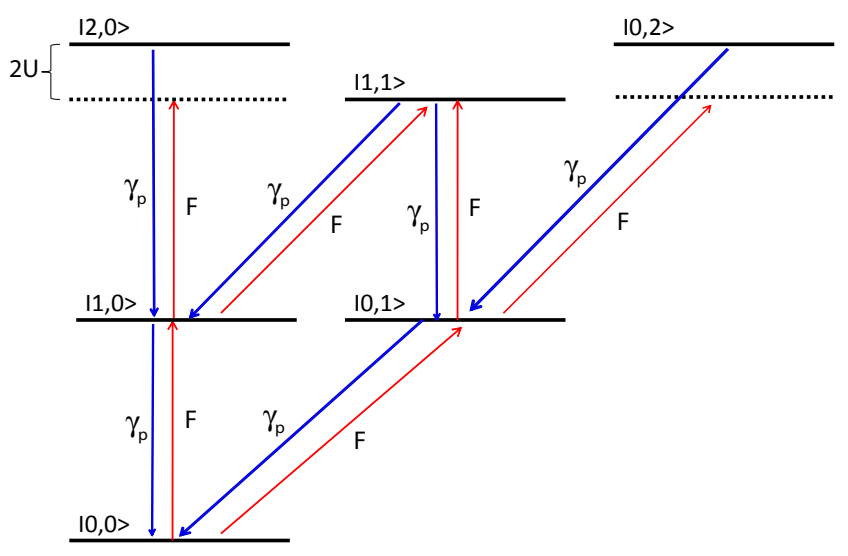

Figure 2: (Color online) Schematic energy level diagram and rates for the coupled cavity system. We use the following shorthand notation for the eigenstates: $|0,0\rangle \rightarrow|1\rangle,|1,0\rangle \rightarrow$ $|2\rangle,|0,1\rangle \rightarrow|3\rangle,|2,0\rangle \rightarrow|4\rangle,|1,1\rangle \rightarrow|5\rangle,|0,2\rangle \rightarrow|6\rangle$.

\section{RESULTS}

The aim of the present work is to assess the secondorder correlation function as a quantitative probe of the interplay between tunneling and interactions in a twosite CQED system described by Eq. 77). To this end, we calculate the steady state normalized degree of secondorder coherence,, 58 i.e. $g_{s s}^{(2)}(\tau)$, for the light emitted from the system, which is defined as

$g_{s s}^{(2)}(\tau)=g^{(2)}(t \rightarrow \infty, \tau)=\frac{\left\langle\hat{p}^{\dagger}(t) \hat{p}^{\dagger}(t+\tau) \hat{p}(t+\tau) \hat{p}(t)\right\rangle}{\left\langle\hat{p}^{\dagger}(t) \hat{p}(t)\right\rangle^{2}}$

In the following, we will only be concerned with the zerotime delay correlation function in steady state, $g_{s s}^{(2)}(0)=$ $\left\langle\hat{p}^{\dagger} \hat{p}^{2}\right\rangle /\left\langle\hat{p}^{\dagger} \hat{p}\right\rangle^{2}$. This correlation function of the polaritons can be straightforwardly related to the correlation function of cavity photons via Eqs. (4), which is the quantity ultimately measured in a typical experiment.

In the weak pumping limit, an analytic solution to the steady state master equation for our model can be found in the following way. We write Eqs. (7) and (9) in the Fock basis $\left\{\left|n_{1}, n_{2}\right\rangle\right\}$, where $n_{1}$ and $n_{2}$ indicate polariton occupations in cavities 1 and 2, respectively. We consider the low energy excitations of the Hamiltonian (8), $N_{t o t}=$ $n_{1}+n_{2} \leq 2$. The corresponding energy level diagram and rates are schematically shown in Fig. 2. The resulting equations of motion for the 36 elements of the density matrix can be solved using perturbation theory and a recursive procedure in $F / \gamma_{p}$, as described in App. A.

The steady-state second-order correlation function 
$g_{s s}^{(2)}(0)$ for the $i$ th cavity can be calculated as

$g_{i}^{(2)}(0)=\frac{\operatorname{Tr}\left\{\hat{p}_{i}^{\dagger} \hat{p}_{i}^{\dagger} \hat{p}_{i} \hat{p}_{i} \rho_{\mathrm{ss}}\right\}}{\left[\operatorname{Tr}\left\{\hat{p}_{i}^{\dagger} \hat{p}_{i} \rho_{\mathrm{ss}}\right\}\right]^{2}}=\frac{\sum_{m m^{\prime}} \rho_{m, m^{\prime}}^{\mathrm{ss}}\left\langle m\left|\hat{p}_{i}^{\dagger} \hat{p}_{i}^{\dagger} \hat{p}_{i} \hat{p}_{i}\right| m^{\prime}\right\rangle}{\left[\sum_{m, m^{\prime}} \rho_{m, m^{\prime}}^{\mathrm{ss}}\left\langle m\left|\hat{p}_{i}^{\dagger} \hat{p}_{i}\right| m^{\prime}\right\rangle\right]^{2}}$,

where $|m\rangle \equiv\left|n_{1} n_{2}\right\rangle$ is a collective notation for the eigenstates of the coupled cavity system and $\rho_{m, m^{\prime}}^{\mathrm{ss}}$ is the steady-state density matrix calculated from Eqs. (7). We will henceforth drop the subscript "ss". In the weak pumping limit $F / \gamma_{p} \ll 1$

$$
g_{1}^{(2)}(0)=g_{2}^{(2)}(0)=g^{(2)}(0) \cong \frac{2 \rho_{4,4}}{\rho_{2,2}^{2}} .
$$

The calculations are lengthy but straightforward (see App. A), and the analytic expressions for the matrix elements $\rho_{4,4}$ and $\rho_{2,2}$ are given in Eqs. A49 and (A29, respectively. Notice that the explicit analytic expression for $\rho_{4,4}$ is recursively obtained through the analytic expression for the elements in Eqs. A29, A33, A34, A41, and A42. The procedure can be generalized, e.g., to perturbatively calculate $g^{(2)}(0)$ for generic multisite CQED systems, either analytically or numerically through the implementation of this recursive algorithm. We notice that setting $J=0$ in the above elements, Eq. (12) gives the correct limit of the single cavity photon blockade

$$
g^{(2)}(0) \cong \frac{1}{1+4\left(U / \gamma_{p}\right)^{2}}
$$

For $J \neq 0$, the resulting behavior of $g^{(2)}(0)$ is shown in Fig. 3 as a function of dimensionless quantities $U / \gamma_{p}$ and $J / \gamma_{p}$. The most striking feature of this plot is the sharp boundary that divides regions where $g^{(2)}(0) \approx 0$ and $g^{(2)}(0) \approx 1$. As clearly seen in Fig. 3, when the tunneling term dominates over the on-site interaction energy the emitted light is Poissonian, $g^{(2)}(0) \approx 1$. This reflects the statistics of the coherent driving fields imposed on the system due to the dominance of the linear tunneling terms over the non-linear interaction terms in the Hamiltonian. Hence the system state is coherently delocalized over both cavities with symmetric and antisymmetric combinations of the bare polariton modes. In the opposite limit, the emitted photons are antibunched, $g^{(2)}(0) \approx 0$, which is a clear indication that the number of quasi-particles in each cavity can only fluctuate between zero and one. The latter is the photon-blockade regime that would be present for $J=0$ (i.e. individual cavities) for $U \gg \gamma_{p}$ (See Eq. (13)). Note that for $J \neq 0$ the cross-over takes place for larger values of $U$ as $J$ is increased, thus showing the interplay of tunneling and interactions in the steady state. The boundary of the crossover in $U, U_{b}(J)$, can be clearly identified following the $g^{(2)}(0)=0.5$ contour (see red dashed line in the plot) and is found to increase approximately linearly with $J$ for $J, U>\gamma_{p}$

In order to check the accuracy of the analytical solution presented above, we have numerically solved Eqs. (7)- 9 .
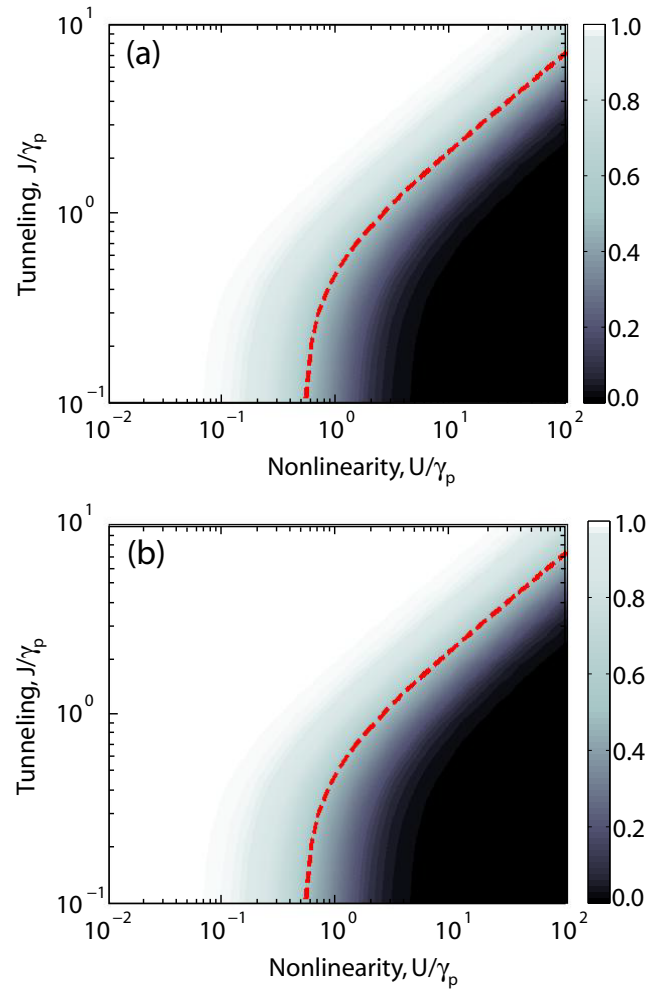

Figure 3: (Color online) The second-order correlation function for the two coupled nonlinear cavities, $g^{(2)}(0)$, as a function of $U / \gamma_{p}$ and $J / \gamma_{p}$ (a) as derived analytically from Eq. (12) and (b) from numerical solution of Eq. 7, calculated for $F / \gamma_{p}=$ 0.1 . The dashed lines in both (a) and (b) show the boundary curve $U_{b}(J)$ defined by the condition $2 \rho_{4,4} / \rho_{2,2}^{2}=0.5$.

The second order correlation function is numerically calculated using Eq. (11), by using up to 6 photons in the Fock basis to ensure full convergence (a small $F / \gamma_{p}$ is assumed to compare with the perturbative analytic solution). The result is shown in Fig. 3(b) with a color scale plot, displaying $g^{(2)}(0)$ as a function of $U / \gamma_{p}$ and $J / \gamma_{p}$ to be directly compared to Fig. 3(a). To better show the agreement and the accuracy of the analytical procedure provided in this work, we give in Fig. 4 several cuts of the color plots of Fig. 3. The quantitative behavior of $g^{(2)}(0)$ is very well reproduced by the analytic solution over several decades considered in the parameter space.

\section{PHYSICAL REALIZATION}

The Kerr-Hubbard Hamiltonian Eq. (1) can be realized with a number of systems, including strongly-coupled atom-cavity systems, 28 circuit QED systems, $\frac{37}{33}$ or quantum dots coupled to semiconductor resonators. 

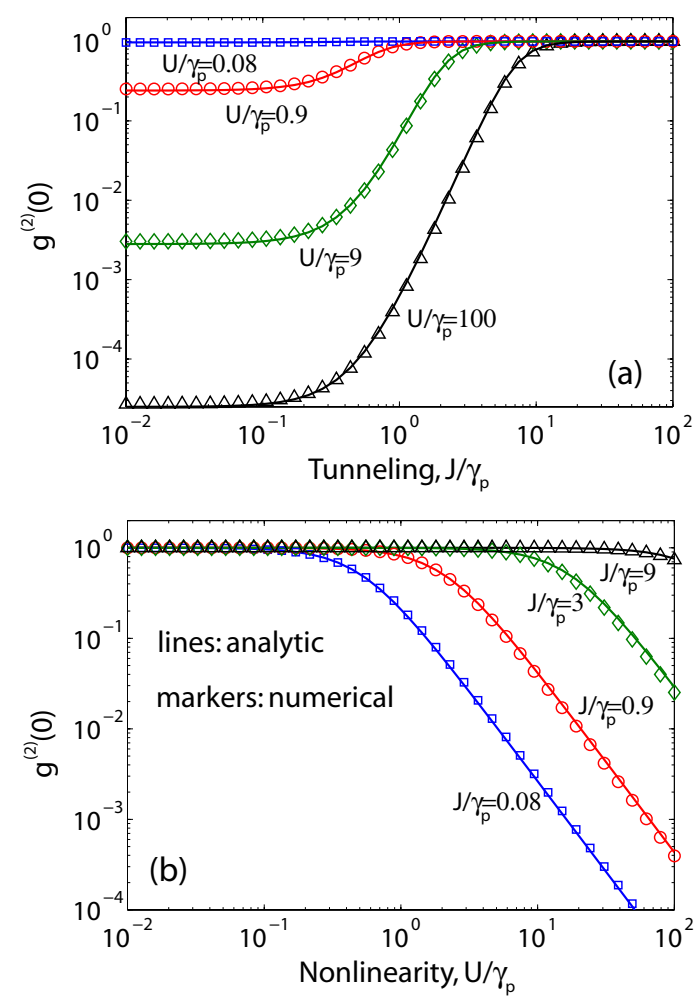

Figure 4: (Color online) A direct comparison of numerical and analytic results for $g^{(2)}(0)$ : (a) fixed $U / \gamma_{p}$ and varying $J / \gamma_{p}$, and (b) fixed $J / \gamma_{p}$ and varying $U / \gamma_{p}$. The full lines represent the analytic solutions, while numerical ones are shown by symbols.

Here, we discuss possible realization with semiconductor-based optical microcavities. Efficient coupling of optical modes in photonic resonators in evanescent contact with each other has been already demonstrated in a number of different systems, including micro-pillars, $\frac{59}{5}$ micro-disks, $\frac{60}{6}$ and photonic crystal cavities. $\frac{52153}{5}$ For the latter, the constant improvement of post-fabrication techniques that allow to deterministically tune the cavity modes ${ }^{6162}$ as well as their coupling to realize the regime of symmetric sites studied here. Note that the tolerance in the parameters for observing qualitatively the phenomena studied here in the symmetric limit is given by the loss rate $\gamma_{p}$. A wide range of tunability has been experimentally demonstrated in such systems, e.g. for tunnel coupling rates $(J \sim 0-1 \mathrm{meV}), \frac{53}{5}$ and Q-factor engineering allows cavity loss rates to be limited to few $\mu \mathrm{eV}$.63

Concerning the nonlinearity, as we have seen in Sec. II the effective Kerr-Hubbard model can be realized either with strongly coupled two-level systems (qubits) and cavity modes, or through coupled light-matter states (po- laritons) with contact-type interaction of Coulomb nature. In the first case, sizeable values of the nonlinearity have been already shown experimentally in solid-state systems, $1113 \mid 17$ and tunability might be achieved through external control of the qubit-cavity detuning (see, e.g., Supplementary Information Section of Ref. 51). In the second case, for which photonic crystals represent an ideal platform for a prospective implementation, 64 very large values of exciton-exciton interactions in confined systems have been predicted for single resonators, ${ }^{22}$ and experimentally measured recently $\left[{ }^{65}\right.$

Finally, detection of emitted radiation and subsequent measurement of correlation functions would be performed by standard Hanbury-Brown-Twiss technique, details depending on the geometry of the system under consideration. For example, in the case of photonic crystal slab cavity systems emitted light might either be guided through access waveguides and then detected in an "in-plane" geometry, or directly imaged in a camera above the slab as in usual microphotoluminescence experiments. Recently, improved farfield collection from high-Q photonic crystal cavity modes has been reported, ${ }^{66}$ which might also be crucial for the present proposal.

\section{CONCLUSIONS}

The two-site Kerr-Hubbard model represents a paradigmatic system to investigate the interplay of tunneling and strong correlations in a CQED system driven out-of-equilibrium. We have analyzed this system under coherent drive and dissipation and showed that in the weak-pumping limit a simple perturbative-recursive approach can be used to analytically compute the system density matrix elements as well as correlation functions. Such procedure can in principle be generalized to CQED arrays with more than two sites. The analytical solution was shown to be highly accurate over several decades of system parameters by comparing to the numerical solution of the master equation for the system density matrix.

Our results show that the zero-delay second-order correlation function provides an effective probe that can discriminate between coherent and strongly correlated regimes of a two-site CQED system. An interesting extension of the present work is the generalization of the techniques and concepts used here to larger multi-site systems.

\section{Acknowledgments}

We thank R. Fazio and S. Schmidt for discussions. This work was supported from Fondazione Cariplo under grant n. 2007-5259. H.E.T. acknowledges support from the Swiss NSF under Grant No. PP00P2-123519/1. 


\section{Appendix A: Density matrix elements}

We provide below the analytic solution of Eqs. (7)-(9) in the small $F / \gamma_{p}$ limit, where we can truncate the polaritonic Hilbert space to $N_{t o t}=2$ and assume that the vacuum state to have approximately unit occupancy, i.e. $\rho_{1,1} \simeq 1$. The rest of the density matrix elements in steady state (see caption to Fig. 2 for the labelling of the basis states) is given below:

$$
\begin{aligned}
& \rho_{1,1} \simeq 1, \\
& \rho_{1,2}=\rho_{2,1}^{*}=\frac{2 \mathrm{i}}{\gamma_{p}}\left[J \rho_{1,3}+F\left(\sqrt{2} \rho_{1,4}+\rho_{1,1}+\rho_{1,5}-\rho_{2,2}-\rho_{3,2}\right)\right]+2 \sqrt{2} \rho_{2,4}+2 \rho_{3,5}, \\
& \rho_{1,3}=\rho_{3,1}^{*}=\frac{2 \mathrm{i}}{\gamma_{p}}\left[J \rho_{1,2}+F\left(\rho_{1,5}+\sqrt{2} \rho_{1,6}+\rho_{1,1}-\rho_{2,3}-\rho_{3,3}\right)\right]+2 \rho_{2,5}+2 \sqrt{2} \rho_{3,6}, \\
& \rho_{1,4}=\rho_{4,1}^{*}=\frac{\mathrm{i}}{\gamma_{p}-2 \mathrm{i} U}\left[\sqrt{2} J \rho_{1,5}+F\left(\sqrt{2} \rho_{1,2}-\rho_{2,4}-\rho_{3,4}\right)\right], \\
& \rho_{1,5}=\rho_{5,1}^{*}=\frac{i}{\gamma_{p}}\left[\sqrt{2} J\left(\rho_{1,4}+\rho_{1,6}\right)+F\left(\rho_{1,3}+\rho_{1,2}-\rho_{2,5}-\rho_{3,5}\right)\right], \\
& \rho_{1,6}=\rho_{6,1}^{*}=\frac{\mathrm{i}}{\gamma_{p}-2 \mathrm{i} U}\left[\sqrt{2} J \rho_{1,5}+F\left(\sqrt{2} \rho_{1,3}-\rho_{2,6}-\rho_{3,6}\right)\right], \\
& \rho_{2,2}=\frac{i}{\gamma_{p}}\left[J\left(\rho_{2,3}-\rho_{3,2}\right)+F\left(\sqrt{2} \rho_{2,4}+\rho_{2,1}+\rho_{2,5}-\rho_{1,2}-\sqrt{2} \rho_{4,2}-\rho_{5,2}\right)\right]+2 \rho_{4,4}+\rho_{5,5}, \\
& \rho_{2,3}=\rho_{3,2}^{*}=\frac{i}{\gamma_{p}}\left[J\left(\rho_{2,2}-\rho_{3,3}\right)+F\left(\sqrt{2} \rho_{2,6}+\rho_{2,1}+\rho_{2,5}-\rho_{1,3}-\sqrt{2} \rho_{4,3}-\rho_{5,3}\right)\right]+2 \rho_{4,4}+\rho_{5,5} \text {, } \\
& \rho_{3,3}=\frac{i}{\gamma_{p}}\left[J\left(\rho_{3,2}-\rho_{2,3}\right)+F\left(\sqrt{2} \rho_{3,6}+\rho_{3,1}+\rho_{3,5}-\rho_{1,3}-\sqrt{2} \rho_{6,3}-\rho_{5,3}\right)\right]+2 \rho_{4,4}+\rho_{5,5}, \\
& \rho_{2,4}=\rho_{4,2}^{*}=\frac{\mathrm{i}}{\frac{3}{2} \gamma_{p}-2 \mathrm{i} U}\left[J\left(\sqrt{2} \rho_{2,5}-\rho_{3,4}\right)+F\left(\sqrt{2} \rho_{2,2}-\rho_{1,4}-\sqrt{2} \rho_{4,4}-\rho_{5,4}\right)\right], \\
& \rho_{2,5}=\rho_{5,2}^{*}=\frac{2}{3} \frac{i}{\gamma_{p}}\left[J\left(\sqrt{2} \rho_{2,4}+\sqrt{2} \rho_{2,6}-\rho_{3,5}\right)+F\left(\rho_{2,3}+\rho_{2,2}-\rho_{1,5}-\sqrt{2} \rho_{4,5}-\rho_{5,5}\right)\right], \\
& \rho_{2,6}=\rho_{6,2}^{*}=\frac{\mathrm{i}}{\frac{3}{2} \gamma_{p}-2 \mathrm{i} U}\left[J\left(\sqrt{2} \rho_{2,5}-\rho_{3,6}\right)+F\left(\sqrt{2} \rho_{2,3}-\rho_{1,6}-\sqrt{2} \rho_{4,6}-\rho_{5,6}\right)\right], \\
& \rho_{3,4}=\rho_{4,3}^{*}=\frac{\mathrm{i}}{\frac{3}{2} \gamma_{p}-2 \mathrm{i} U}\left[J\left(\sqrt{2} \rho_{3,5}-\rho_{2,4}\right)+F\left(\sqrt{2} \rho_{3,2}-\rho_{5,4}-\rho_{1,4}-\sqrt{2} \rho_{6,4}\right)\right], \\
& \rho_{3,5}=\rho_{5,3}^{*}=\frac{2}{3} \frac{\mathrm{i}}{\gamma_{p}}\left[J\left(\sqrt{2} \rho_{3,4}+\sqrt{2} \rho_{3,6}-\rho_{2,5}\right)+F\left(\rho_{3,3}+\rho_{3,2}-\rho_{5,5}-\rho_{1,5}-\sqrt{2} \rho_{6,5}\right)\right], \\
& \rho_{3,6}=\rho_{6,3}^{*}=\frac{\mathrm{i}}{\frac{3}{2} \gamma_{p}-2 \mathrm{i} U}\left[J\left(\sqrt{2} \rho_{3,5}-\rho_{2,6}\right)+F\left(\sqrt{2} \rho_{3,3}-\rho_{5,6}-\sqrt{2} \rho_{6,6}-\rho_{1,6}\right)\right], \\
& \rho_{4,4}=\frac{i}{2 \gamma_{p}}\left[\sqrt{2} J\left(\rho_{4,5}-\rho_{5,4}\right)+\sqrt{2} F\left(\rho_{4,2}-\rho_{2,4}\right)\right] \text {, } \\
& \rho_{4,5}=\rho_{5,4}^{*}=\frac{\mathrm{i}}{2 \gamma_{p}+2 \mathrm{i} U}\left[\sqrt{2} J\left(\rho_{4,4}+\rho_{4,6}-\rho_{5,5}\right)+F\left(\rho_{4,3}+\rho_{4,2}-\sqrt{2} \rho_{2,5}\right)\right] \text {, } \\
& \rho_{4,6}=\rho_{6,4}^{*}=\frac{i}{2 \gamma_{p}}\left[\sqrt{2} J\left(\rho_{4,5}-\rho_{5,6}\right)+\sqrt{2} F\left(\rho_{4,3}-\rho_{2,6}\right)\right], \\
& \rho_{5,5}=\frac{i}{2 \gamma_{p}}\left[\sqrt{2} J\left(\rho_{5,4}+\rho_{5,6}-\rho_{6,5}-\rho_{4,5}\right)+F\left(\rho_{5,3}+\rho_{5,2}-\rho_{3,5}-\rho_{2,5}\right)\right], \\
& \rho_{5,6}=\rho_{6,5}^{*}=\frac{\mathrm{i}}{2 \gamma_{p}-2 \mathrm{i} U}\left[\sqrt{2} J\left(\rho_{5,5}-\rho_{6,6}-\rho_{4,6}\right)+F\left(\sqrt{2} \rho_{5,3}-\rho_{3,6}-\rho_{2,6}\right)\right], \\
& \rho_{6,6}=\frac{i}{2 \gamma_{p}}\left[\sqrt{2} J\left(\rho_{6,5}-\rho_{5,6}\right)+\sqrt{2} F\left(\rho_{6,3}-\rho_{3,6}\right)\right],
\end{aligned}
$$

We simplify the equations above as follows. First we consider the equations with a first order dependence on $F / \gamma_{p}$, and we neglect all the higher order terms. We get

$$
\rho_{1,2}=\rho_{2,1}^{*}=\frac{2 \mathrm{i}}{\gamma_{p}}\left(J \rho_{1,3}+F \rho_{1,1}\right),
$$

and

$$
\rho_{1,3}=\rho_{3,1}^{*}=\frac{2 \dot{i}}{\gamma_{p}}\left(J \rho_{1,2}+F \rho_{1,1}\right) .
$$

This yields

$$
\rho_{1,2}=\rho_{1,3}=\rho_{2,1}^{*}=\rho_{3,1}^{*}=\frac{\frac{2 \mathrm{i}}{\gamma_{p}}\left(1+\frac{2 \mathrm{i} J}{\gamma_{p}}\right)}{1+\left(\frac{2 J}{\gamma_{p}}\right)^{2}} F \rho_{1,1} .
$$

Next we insert these expressions into the remaining equations to obtain equations at a higher order in $F / \gamma_{p}$. Thus, 
the elements of $\rho$ with a second order dependence on $F / \gamma_{p}$ give the following set of closed equations

$$
\begin{aligned}
& \rho_{2,2}=\frac{i}{\gamma_{p}}\left[J\left(\rho_{2,3}-\rho_{3,2}\right)+F\left(\rho_{2,1}-\rho_{1,2}\right)\right]=\frac{i}{\gamma_{p}} F\left(\rho_{2,1}-\rho_{1,2}\right) \\
& \rho_{2,3}=\frac{i}{\gamma_{p}}\left[J\left(\rho_{2,2}-\rho_{3,3}\right)+F\left(\rho_{2,1}-\rho_{1,3}\right)\right]=\frac{i}{\gamma_{p}} F\left(\rho_{2,1}-\rho_{1,3}\right) \\
& \rho_{3,2}=\frac{i}{\gamma_{p}}\left[J\left(\rho_{3,3}-\rho_{2,2}\right)+F\left(\rho_{3,1}-\rho_{1,2}\right)\right]=\frac{i}{\gamma_{p}} F\left(\rho_{3,1}-\rho_{1,2}\right) \\
& \rho_{3,3}=\frac{i}{\gamma_{p}}\left[J\left(\rho_{3,2}-\rho_{2,3}\right)+F\left(\rho_{3,1}-\rho_{1,3}\right)\right]=\frac{i}{\gamma_{p}} F\left(\rho_{3,1}-\rho_{1,3}\right),
\end{aligned}
$$

from which we can calculate a solution in terms of $\rho_{1,1}$ as

$$
\rho_{2,2}=\rho_{2,3}=\rho_{3,2}=\rho_{3,3}=\frac{4}{1+\left(\frac{2 J}{\gamma_{p}}\right)^{2}}\left(\frac{F}{\gamma_{p}}\right)^{2} \rho_{1,1} .
$$

Now we consider the equations of order $\left(F / \gamma_{p}\right)^{3}$

$$
\begin{aligned}
\rho_{1,4} & =\rho_{4,1}^{*}=\frac{\sqrt{2} \mathrm{i} J}{\gamma_{p}-2 \mathrm{i} U} \rho_{1,5}+\frac{\sqrt{2} \mathrm{i} F}{\gamma_{p}-2 \mathrm{i} U} \rho_{1,2} \\
\rho_{1,6} & =\rho_{6,1}^{*}=\frac{\sqrt{2} \mathrm{i} J}{\gamma_{p}-2 \mathrm{i} U} \rho_{1,5}+\frac{\sqrt{2} \mathrm{i} F}{\gamma_{p}-2 \mathrm{i} U} \rho_{1,3} \\
\rho_{1,5} & =\rho_{5,1}^{*}=2 \sqrt{2} \dot{\mathrm{i}} \frac{J}{\gamma_{p}} \rho_{1,4}+2 \mathrm{i} \frac{F}{\gamma_{p}} \rho_{1,2} .
\end{aligned}
$$

Again, we can solve the set of coupled equations reported above isolating the explicit dependence on $\rho_{1,1}$, which gives the solutions

$$
\begin{gathered}
\rho_{1,4}=\rho_{1,6}=\rho_{4,1}^{*}=\rho_{6,1}^{*}=\frac{-2 \sqrt{2}\left(1+\frac{2 \mathrm{i} J}{\gamma_{p}}\right)^{2}}{\left[\gamma_{p}\left(\gamma_{p}-2 \mathrm{i} U\right)+4 J^{2}\right]\left[1+\left(\frac{2 J}{\gamma_{p}}\right)^{2}\right]} F^{2} \rho_{1,1}, \\
\rho_{1,5}=\rho_{5,1}^{*}=-\frac{4 \mathrm{i} J+2\left(\gamma_{p}-2 \mathrm{i} U\right)}{4 J^{2}+\gamma_{p}\left(\gamma_{p}-2 \mathrm{i} U\right)} \frac{\frac{2}{\gamma_{p}}\left(1+\frac{2 \mathrm{i} j}{\gamma_{p}}\right)}{1+\left(\frac{2 J}{\gamma_{p}}\right)^{2}} F^{2} \rho_{1,1} .
\end{gathered}
$$

We analyse the other set of equations of order $\left(F / \gamma_{p}\right)^{3}$

$$
\begin{gathered}
\rho_{2,4}=\frac{\mathrm{i}}{\frac{3}{2} \gamma_{p}-2 \mathrm{i} U}\left[J\left(\sqrt{2} \rho_{2,5}-\rho_{2,6}\right)+F\left(\sqrt{2} \rho_{2,2}-\rho_{1,4}\right)\right], \\
\rho_{2,6}=\frac{\mathrm{i}}{\frac{3}{2} \gamma_{p}-2 \mathrm{i} U}\left[J\left(\sqrt{2} \rho_{2,5}-\rho_{3,6}\right)+F\left(\sqrt{2} \rho_{2,3}-\rho_{1,6}\right)\right], \\
\rho_{3,4}=\frac{\mathrm{i}}{\frac{3}{2} \gamma_{p}-2 \mathrm{i} U}\left[J\left(\sqrt{2} \rho_{3,5}-\rho_{2,4}\right)+F\left(\sqrt{2} \rho_{3,2}-\rho_{1,4}\right)\right], \\
\rho_{3,6}=\frac{\mathrm{i}}{\frac{3}{2} \gamma_{p}-2 \mathrm{i} U}\left[J\left(\sqrt{2} \rho_{3,5}-\rho_{2,6}\right)+F\left(\sqrt{2} \rho_{3,3}-\rho_{1,6}\right)\right], \\
\rho_{2,5}=\frac{2}{3} \frac{\mathrm{i}}{\gamma_{p}}\left[J\left(\sqrt{2} \rho_{2,4}+\sqrt{2} \rho_{2,6}-\rho_{3,5}\right)+F\left(\rho_{2,3}+\rho_{2,2}-\rho_{1,5}\right)\right], \\
\rho_{3,5}=\frac{2}{3} \frac{i}{\gamma_{p}}\left[J\left(\sqrt{2} \rho_{3,4}+\sqrt{2} \rho_{3,6}-\rho_{2,5}\right)+F\left(\rho_{3,3}+\rho_{3,2}-\rho_{1,5}\right)\right],
\end{gathered}
$$

and we get a solution depending on the elements $\rho_{2,2}, \rho_{1,4}, \rho_{1,5}$, which in turn depend on $\rho_{1,1}$ as shown above. The solutions for $\rho_{2,4}$ and $\rho_{2,5}$ read

$$
\begin{gathered}
\rho_{2,4}=\rho_{3,6}=\rho_{2,6}=\rho_{3,4}=\rho_{4,2}^{*}=\rho_{6,3}^{*}=\rho_{6,2}^{*}=\rho_{4,3}^{*}=\mathrm{i} F \frac{\left(3 \gamma_{p}+2 \mathrm{i} J\right)\left(\sqrt{2} \rho_{2,2}-\rho_{1,4}\right)+2 \sqrt{2} \mathrm{i} J\left(2 \rho_{2,2}-\rho_{1,5}\right)}{\left(\frac{3}{2} \gamma_{p}-2 \mathrm{i} U\right)\left(3 \gamma_{p}+2 \mathrm{i} J\right)+3 \mathrm{i} \gamma_{p} J+6 J^{2}} \\
\rho_{2,5}=\rho_{3,5}=\rho_{5,2}^{*}=\rho_{5,3}^{*}=\mathrm{i} F \frac{4 \sqrt{2} \mathrm{i} J\left(\sqrt{2} \rho_{2,2}-\rho_{1,4}\right)+2\left[\left(\frac{3}{2} \gamma_{p}-2 \mathrm{i} U\right)+\mathrm{i} J\right]\left(2 \rho_{2,2}-\rho_{1,5}\right)}{\left(\frac{3}{2} \gamma_{p}-2 \mathrm{i} U\right)\left(3 \gamma_{p}+2 \mathrm{i} J\right)+3 \mathrm{i} \gamma_{p} J+6 J^{2}}
\end{gathered}
$$

We finally consider the terms depending on $\left(F / \gamma_{p}\right)^{4}$,

$$
\begin{aligned}
\rho_{4,5}=\rho_{5,4}^{*} & =\frac{i}{2 \gamma_{p}+2 \mathrm{i} U}\left[\sqrt{2} J\left(2 \rho_{4,4}-\rho_{5,5}\right)+F\left(2 \rho_{4,2}-\sqrt{2} \rho_{2,5}\right)\right], \\
\rho_{5,5} & =\frac{i}{\gamma_{p}}\left[\sqrt{2} J\left(\rho_{5,4}-\rho_{4,5}\right)+F\left(\rho_{5,2}-\rho_{2,5}\right)\right], \\
\rho_{4,4} & =\frac{i}{2 \gamma_{p}}\left(\sqrt{2} J\left(\rho_{4,5}-\rho_{5,4}\right)+\sqrt{2}\left(\rho_{4,2}-\rho_{2,4}\right),\right. \\
\rho_{4,6} & =\frac{i}{2 \gamma_{p}}\left(\sqrt{2} J\left(\rho_{4,5}-\rho_{5,6}\right)+\sqrt{2}\left(\rho_{4,3}-\rho_{2,6}\right),\right. \\
\rho_{6,4} & =\frac{i}{2 \gamma_{p}}\left(\sqrt{2} J\left(\rho_{6,5}-\rho_{5,4}\right)+\sqrt{2}\left(\rho_{6,2}-\rho_{3,4}\right),\right. \\
\rho_{6,6} & =\frac{i}{2 \gamma_{p}}\left(\sqrt{2} J\left(\rho_{6,5}-\rho_{5,6}\right)+\sqrt{2}\left(\rho_{6,3}-\rho_{3,6}\right) .\right.
\end{aligned}
$$


from these closed set of equations we get a solution for $\rho_{4,4}$ as

$$
\begin{gathered}
\rho_{4,4}=\rho_{6,6}=\rho_{4,6}=\rho_{6,4}= \\
\frac{F}{2 \gamma_{p}\left[\left(2 \gamma_{p}\right)^{2}+(2 U)^{2}+(4 J)^{2}\right]} \times\left[\Im\left(\rho_{2,4}\right) 8 \sqrt{2}\left(\gamma_{p}^{2}+U^{2}+2 J^{2}+J U\right)+-\Re\left(\rho_{2,4}\right) 8 \sqrt{2} \gamma_{p} J+\right. \\
\left.+\Im\left(\rho_{2,5}\right) 8 J(2 J+U)+\Re\left(\rho_{2,5}\right) 8 \gamma_{p} J\right],
\end{gathered}
$$

where $\Re$ and $\Im$ indicate real and imaginary parts of the respective elements. The explicit analytic expression for $\rho_{4,4}$ can be calculated from those of the elements $\rho_{2,2}, \rho_{1,4}, \rho_{1,5}, \rho_{2,4}, \rho_{2,5}$, which have been obtained before. We notice that the recursive procedure described can be properly generalized to calculate the relevant density matrix elements also for generic multi-site CQED systems.

\section{Appendix B: Numerical solution}

To solve Eqs. (7)-(9) we use the finite-size Fock-state matrix representation of all the operators. For any given set of model parameters, the steady state density matrix can be obtained by numerically searching for the eigenvector $|\rho\rangle\rangle_{s s}$ corresponding to the eigenvalue $\lambda_{s s}=0$ of the linear operator equation

$$
\hat{L}|\rho\rangle\rangle=\lambda|\rho\rangle\rangle .
$$

In the latter, $|\rho\rangle\rangle$ is the density operator mapped into vectorial form, and $\hat{L}$ is the linear matrix corresponding to the Liouvillian superoperator on the right-hand side of the master equation. If it exists, as it is always the case for the parameters considered, the steady state solution is unique. ${ }^{67}$ After recasting the vector $\left.|\rho\rangle\right\rangle_{s s}$ in matrix form, the relevant observable quantities can be calculated as $\langle O\rangle_{s s}=\operatorname{Tr}\left\{\hat{O} \rho_{s s}\right\}$. In this work, we kept up to 6 photons per cavity in the basis, which is sufficient for convergence due to the weak driving conditions. Steady state results obtained in this way have been successfully compared to the ones obtained from a full time evolution of Eq. (7) as a further check.

1 J. M. Raimond, M. Brune, and S. Haroche, Rev. Mod. Phys. 73, 565 (2001).

${ }^{2}$ H. Mabuchi and A. C. Doherty, Science 298, 1372 (2002).

3 S. Haroche and J.-M. Raimond, Exploring the Quantum: Atoms, Cavities and Photons (Oxford University Press, 2006).

${ }^{4}$ E. T. Jaynes and F. W. Cummings Proc. IEEE 51, 89 (1963).

${ }^{5}$ H. J. Carmichael, R. J. Brecha, M. G. Raizen, H. J. Kimble, and P. R. Rice, Phys. Rev. A 40, 5516 (1989).

6 L. C. Andreani, G. Panzarini, and J.-M. Gérard, Phys. Rev. B 60, 13276 (1999).

7 J. P. Reithmaier, G. Sek, A. Löffler, C. Hofmann, S. Kuhn, S. Reitzenstein, L. V. Keldysh, V. D. Kulakovkii, T. L. Reinecke, and A. Forchel, Nature (London) 432, 197 (2004); T. Yoshie, A. Scherer, J. Hendrickson, G. Khitrova, H. M. Gibbs, G. Rupper, C. Ell, O. B. Shchekin, and D. G. Deppe, Nature (London) 432, 200 (2004); E. Peter, P. Senellart, D. Martrou, A. Lemaitre, J. Hours, J.-M. Gérard, and J. Bloch, Phys. Rev. Lett. 95, 067401 (2005).

8 A. Wallraff, D. I. Schuster, A. Blais, L. Frunzio, R. S. Huang, J. Majer, S. Kumar, S. M. Girvin, and R. J. Schoelkopf, Nature (London) 431, 162 (2004).

9 Q. A. Turchette, C. J. Hood, W. Lange, H. Mabuchi, and H. J. Kimble, Phys. Rev. Lett. 75, 4710 (1995).

10 I. Schuster, A. Kubanek, A. Fuhrmanek, T. Puppe, P. W. H. Pinkse, K. Muss, and G. Rempe, Nat. Physics 4, 382
(2008).

11 K. Hennessy, A. Badolato, M. Winger, D. Gerace, M. Atatüre, S. Gulde, S. Fält, E. Hu, and A. Imamoğlu, Nature (London) 445, 896 (2007).

12 K. Srinivasan and O. Painter, Nature (London) 450, 862 (2007).

13 L. S. Bishop, J. M. Chow, J. Koch, A. A. Houck, M. H. Devoret, E. Thuneberg, S. M. Girvin, R. J. Schoelkopf, Nat. Physics 5, 105 (2009).

14 E. del Valle, F. P. Laussy, and C. Tejedor, Phys. Rev. B 79, 235326 (2009).

15 M. J. Werner and A. Imamoğlu, Phys. Rev. A 61, R011801 (1999).

16 K. M. Birnbaum, A. Boca, R. Miller, A. D. Boozer, T. E. Northup, and H. J. Kimble, Nature (London) 436, 87 (2005).

17 A. Faraon, I. Fushman, D. Englund, N. Stoltz, P. Petroff, and J. Vučković, Nat. Physics 4, 859 (2008).

18 I. Carusotto, Phys. Rev. A 63, 23610 (2001).

19 S. Rebić, A. S. Parkins, and S. M. Tan, Phys. Rev. A 69, 035804 (2004).

20 I. Carusotto, T. Volz, and A. Imamoğlu, Europhys. Lett. 90, 37001 (2010).

21 O. El Daif, T. Guillet, A. Baas, J.-P. Brantut, R. Idrissi-Kaitouni, J.-L. Staehli, F. Morier-Genoud, and B. Deveaud-Pledran, Appl. Phys. Lett. 88, 061105 (2006).

22 A. Verger, C. Ciuti, and I. Carusotto, Phys. Rev. B 73, 
193306 (2006).

23 O. El Daif, G. Nardin, T. K. Paraiso, A. Baas, M. Richard, J.-P. Brantut, F. Morier-Genoud, B. Deveaud-Pledran, and T. Guillet, Appl. Phys. Lett. 92, 081910 (2008).

24 M. P. A. Fisher, P. B. Weichman, G. Grinstein, and D. S. Fisher, Phys. Rev. B 40, 546 (1989).

25 D. Jaksch, C. Bruder, J. I. Cirac, C. W. Gardiner, and P. Zoller, Phys. Rev. Lett. 81, 3108 (1998).

26 M. Greiner, O. Mandel, T. Esslinger, T. W. Hänsch, and I. Bloch, Nature (London) 415, 39 (2002).

27 M. J. Hartmann, F. G. S. L. Brandão, and M. B. Plenio, Laser Photonics Rev. 2, 527 (2008).

${ }^{28}$ M. J. Hartmann, F. G. S. L. Brandão, and M. B. Plenio, Nat. Physics 2, 849 (2006).

29 N. Na, S. Utsunomiya, L. Tian, and Y. Yamamoto, Phys. Rev. A 77, 031803(R) (2008).

30 M. Aichhorn, M. Hohenadler, C. Tahan, and P. B. Littlewood, Phys. Rev. Lett. 100, 216401 (2008).

31 A. Kay and D. G. Angelakis, Eur. Phys. Lett. 84, 20001 (2008)

32 P. Pippan, H. G. Evertz, and M. Hohenadler, Phys. Rev. A 80, 033612 (2009).

33 A. D. Greentree, C. Tahan, J. H. Cole, and L. C. L. Hollenberg, Nat. Physics 2, 856 (2006).

34 D. Rossini and R. Fazio, Phys. Rev. Lett. 99, 186401 (2007).

35 M. I. Makin, J. H. Cole, C. Tahan, L. C. L. Hollenberg, and A. D. Greentree, Phys. Rev. A 77, 053819 (2008).

36 M. Grochol, Phys. Rev. B 79, 205306 (2009).

37 J. Koch and K. Le Hur, Phys. Rev. A 80, 023811 (2009).

38 S. Schmidt and G. Blatter, Phys. Rev. Lett. 103, 086403 (2009)

39 S. Schmidt and G. Blatter, Phys. Rev. Lett. 104, 216402 (2010).

40 C. D. Ogden, E. K. Irish, and M. S. Kim, Phys. Rev. A 78, 063805 (2008).

41 S. Schmidt, D. Gerace, A. A. Houck, G. Blatter, and H. E. Türeci, arXiv:1006.0094

42 M. Paternostro, G. S. Agarwal, and M. S. Kim, New J. Phys. 11, 013059 (2009).

43 J. Cho, D. G. Angelakis, and S. Bose, Phys. Rev. Lett. 101, 246809 (2008)

44 D. E. Chang, V. Gritsev, G. Morigi, V. Vuletic, M. D. Lukin, and E. A. Demler, Nat. Physics 4, 884 (2008).

45 I. Carusotto, D. Gerace, H. E. Türeci, S. De Liberato, C. Ciuti, and A. Imamoglu, Phys. Rev. Lett. 103, 033601 (2009).

46 D. G. Angelakis, M. F. Santos, and S. Bose, Phys. Rev. A 76, 031805(R) (2007).

47 M. J. Hartmann, F. G. S. L. Brandão, and M. B. Plenio,
Phys. Rev. Lett. 99, 160501 (2007).

48 J. Cho, D. G. Angelakis, and S. Bose, Phys. Rev. A 78, 022323 (2008).

49 T. C. H. Liew and V. Savona, Phys. Rev. Lett. 104, 183601 (2010).

50 A. Tomadin, V. Giovannetti, R. Fazio, D. Gerace, I. Carusotto, H. E. Türeci, and A. Imamoğlu, Phys. Rev. A 81, 061801(R) (2010)

51 D. Gerace, H. E. Türeci, A. Imamoğlu, V. Giovannetti, and R. Fazio, Nat. Physics 5, 281 (2009).

${ }^{52}$ K. A. Atlasov, K. F. Karlsson, A. Rudra, B. Dwir, and E. Kapon, Opt. Express 16, 16255 (2008).

53 S. Vignolini, F. Intonti, M. Zani, F. Riboli, D. S. Wiersma, L. H. Li, L. Balet, M. Francardi, A. Gerardino, A. Fiore, and M. Gurioli, Appl. Phys. Lett. 94, 151103 (2009).

54 A formal derivation in the strongly dispersive regime, i.e. at large atom-cavity detuning where $\left\langle\sigma_{z}\right\rangle \approx-1$, is discussed, e.g., in M. Boissonneault, J. M. Gambetta, and A. Blais, Phys. Rev. A 79, 013819 (2009).

55 C. Ciuti, P. Schwendimann, and A. Quattropani, Semicond. Sci. Technol. 18, S279 (2003).

56 H. Carmichael, An open systems approach to quantum optics (Springer-Verlag, Berlin, 1993).

57 The polariton dissipation rate can be expressed through Eq. (3) as the weighted sum of the excitonic dissipation $\left(\gamma_{x}\right)$ and bare cavity photon $(\kappa)$ dissipation rates, $\gamma_{p}=$ $u^{2} \gamma_{x}+v^{2} \kappa$.

58 R. Loudon, The quantum theory of light (Oxford University Press, 2003).

59 M. Karl, S. Li, T. Passow, W. Löffler, H. Kalt, and M. Hetterich, Opt. Express 15, 8191 (2007).

${ }^{60}$ M. Benyoucef, S. Kiravittaya, Y. F. Mei, A. Rastelli, and O. G. Schmidt, Phys. Rev. B 77, 035108 (2008).

${ }^{61}$ K. Hennessy, C. Högerle, E. Hu, A. Badolato, and A. Imamoglu, Appl. Phys. Lett. 89, 041118 (2006).

62 H. S. Lee, S. Kiravittaya, S. Kumar, J. D. Plumhof, L. Balet, L. H. Li, M. Francardi, A. Gerardino, A. Fiore, A. Rastelli, O. G. Schmidt, Appl. Phys. Lett. 95, 191109 (2009).

63 S. Combrié, A. De Rossi, N.-Q.-V. Tran, and H. Benisty, Opt. Lett. 33, 1908 (2008).

64 D. Gerace and L. C. Andreani, Phys. Rev. B 75, 235325 (2007).

65 Z. Voros, D. W. Snoke, L. Pfeiffer, and K. West, Phys. Rev. Lett. 103, 016403 (2009).

66 N.-V.-Q. Tran, S. Combrié, and A. De Rossi, Phys. Rev. B 79, 041101(R) (2009).

67 M. Jakob and S. Stenholm, Phys. Rev. A 67, 032111 (2003). 